\title{
Characteristics of angular cross correlations studied by light scattering from two-dimensional microsphere films
}

\author{
M. A. Schroer, ${ }^{*}$ C. Gutt, ${ }^{\dagger}$ and G. Grübel \\ Deutsches Elektronen-Synchrotron DESY, Notkestr. 85, 22607 Hamburg, Germany \\ and The Hamburg Centre for Ultrafast Imaging (CUI), Luruper Chausee 149, 22761 Hamburg, Germany
}

(Received 15 January 2014; published 31 July 2014)

\begin{abstract}
Recently the analysis of scattering patterns by angular cross-correlation analysis (CCA) was introduced to reveal the orientational order in disordered samples with special focus to future applications on $\mathrm{x}$-ray free-electron laser facilities. We apply this CCA approach to ultra-small-angle light-scattering data obtained from two-dimensional monolayers of microspheres. The films were studied in addition by optical microscopy. This combined approach allows to calculate the cross-correlations of the scattering patterns, characterized by the orientational correlation function $\Psi_{l}(q)$, as well as to obtain the real-space structure of the monolayers. We show that CCA is sensitive to the orientational order of monolayers formed by the microspheres which are not directly visible from the scattering patterns. By mixing microspheres of different radii the sizes of ordered monolayer domains is reduced. For these samples it is shown that $\Psi_{l}(q)$ quantitatively describes the degree of hexagonal order of the two-dimensional films. The experimental CCA results are compared with calculations based on the microscopy images. Both techniques show qualitatively similar features. Differences can be attributed to the wave-front distortion of the laser beam in the experiment. This effect is discussed by investigating the effect of different wave fronts on the cross-correlation analysis results. The so-determined characteristics of the cross-correlation analysis will be also relevant for future $\mathrm{x}$-ray-based studies.
\end{abstract}

DOI: 10.1103/PhysRevE.90.012309

PACS number(s): 64.75.Xc, 61.05.cf, 68.37.Yz

\section{INTRODUCTION}

Scattering experiments are powerful tools to resolve the structure of matter. However, to completely determine the microscopic structure of a sample, diffraction data of single crystals or perfectly ordered arrangements of crystals are needed. For disordered samples lacking long-range order, such as liquids, glasses, and amorphous solids, conventional analysis schemes give information on only the radial pair distribution function $g(r)$ via measuring the azimuthally averaged static structure factor $S(q)$ [1-5]. Thus, information on the orientational order within the sample is not accessible. In order to overcome these limitations, angular cross-correlation analysis (CCA) approaches for $\mathrm{x}$-ray-scattering data were introduced recently [6]. With this so-called X-ray crosscorrelation analysis (XCCA) technique the hidden local order in a colloidal glass was made visible. The application of XCCA to detect the local order of disordered material is especially interesting for $\mathrm{x}$-ray free-electron laser (XFEL) facilities [7]. Due to their ultrashort pulse duration these facilities have the potential to study the orientational order within molecular liquids and shed more light into local structures of these. For these types of studies knowledge on the characteristics of this cross-correlation-analysis approach is essential.

The concept of using angular cross correlations was introduced by Kam in the late 1970s [8]. There it was proposed to use angular correlation functions for reconstructing the three-dimensional shape of diluted particles in solutions. Just

*martin.schroer@desy.de

${ }^{\dagger}$ Present address: Universität Siegen, Walter-Flex-Str. 3, 57072 Siegen, Germany. recently this approach was used successfully to experimental data [9-13].

For concentrated particle solutions and amorphous solids characterized by medium-range order the first CCA schemes were employed using light-scattering and electron diffraction data $[14,15]$. Thereafter, the $\mathrm{x}$-ray cross-correlation-analysis approach was explored in more detail theoretically for disordered systems of concentrated objects [16-19]. These recent studies were actually carried out on two-dimensional systems. Therefore, experimental studies on such type of samples will allow a direct comparison with these theories.

The preparation of purely two-dimensional systems on length scales from several to several hundred nanometers needed for transmission $\mathrm{x}$-ray-scattering experiments is hard to achieve. However, micrometer-sized objects and visible light offer the possibility to study diffraction from quasi-twodimensional objects [14]. While the film structure can additionally be probed by optical microscopy, the light-scattering patterns can be analyzed by angular cross-correlation analysis. The so-obtained CCA results can then be directly compared to those from theory.

In the present study we report on a cross-correlation analysis on two-dimensional microsphere films exhibiting hexagonally ordered domains. By employing particles of $\mu \mathrm{m}$ size ultra-small-angle light scattering (USALS) as well as optical microscopy can be used to investigate the sample structure. This combined approach allows us to directly relate the light CCA (LCCA) results to the underlying structure of the dry microsphere film. Our findings can be directly compared to a recent simulation and theoretical study on cross-correlation analysis on hexagonal structures on a grid [19].

First, we introduce the orientational correlation function $\Psi_{l}(q)$ which can be determined from scattering patterns of the sample. To perform the USALS measurements a dedicated 
setup was built up. CCA spectra are obtained from the light-scattering patterns. These spectra exhibit the characteristics of hexagonal order of the microsphere monolayer. The experimental CCA results are compared with those calculated from the structures visible in the micrographs. We investigated several mixtures of microspheres in order to induce disorder in the hexagonal film structure to learn how this affects the LCCA result. It can be shown that $\Psi_{l}(q)$ is a quantitative measure for the degree of hexagonal orientational order within the samples. In addition, by combining the USALS data and micrographs we show that the shape of the wave front of the laser beam has a crucial influence to the CCA spectra. The influence of a nonplanar wave front on $\Psi_{l}(q)$ is discussed. We expect that these findings on the cross-correlation analysis for two-dimensional systems have an influence on future $\mathrm{x}$-ray scattering experiments on synchrotron facilities and XFELs.

\section{THEORY}

In a typical scattering experiment, the scattering intensity $I(\vec{q})$ is recorded as a function of the wave vector transfer whose modulus in case of light scattering is given as $q=$ $\frac{4 \pi n}{\lambda} \sin (\Theta / 2)$. Herein $n$ denotes the index of refraction of the sample, $\lambda$ the wavelength of the light, and $\Theta$ the scattering angle. When employing a two-dimensional detector, the scattering pattern $I(\vec{q})$ is described in terms of $q$ and the azimuthal angle $\phi$, i.e., $I(q, \phi)$.

The general definition of an angular cross-correlation function of such a two-dimensional scattering pattern is [6]

$$
C(q, \Delta)=\frac{\langle I(q, \phi) I(q, \phi+\Delta)\rangle_{\phi}-\langle I(q, \phi)\rangle_{\phi}^{2}}{\langle I(q, \phi)\rangle_{\phi}^{2}},
$$

wherein $\Delta$ specifies the angle between two radial cross sections through the pattern (Fig. 1). $\langle\cdot\rangle_{\phi}$ denotes the angular average. The angular Fourier coefficient of $l$-th order, $\hat{C}_{l}(q)$, is given as

$$
\hat{C}_{l}(q)=\int_{0}^{2 \pi} C(q, \Delta) \exp (i l \Delta) d \Delta .
$$

The $l$-th Fourier coefficient is connected to the normalized angular Fourier coefficient of the scattering intensity, $\hat{I}_{l}^{\mathrm{N}}(q)$, via the Wiener-Khinchin theorem, i.e.,

$$
\hat{C}_{l}(q)=\left|\hat{I}_{l}^{\mathrm{N}}(q)\right|^{2} \text {. }
$$

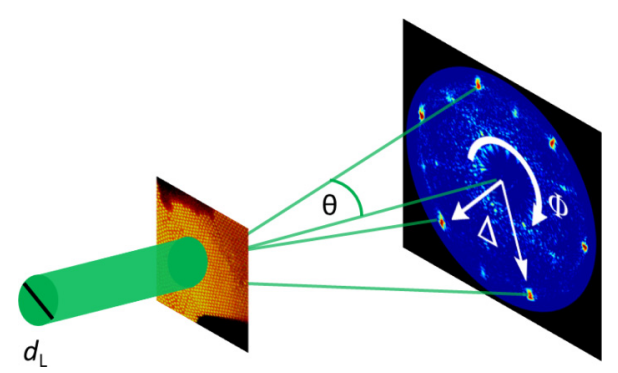

FIG. 1. (Color online) Schematic layout of the scattering experiment on a microsphere film. A laser beam with diameter $d_{\mathrm{L}}$ impinges on the sample and gives rise to a scattering pattern detected by a CMOS-based camera.
With this, the variance of $\hat{I}_{l}^{\mathrm{N}}(q)$ is given as [19]

$$
\Psi_{l}(q):=\operatorname{var}\left(\hat{I}_{l}^{\mathrm{N}}(q)\right)=\left\langle\hat{C}_{l}(q)\right\rangle_{\mathrm{e}}-\left\langle\hat{I}_{l}^{\mathrm{N}}(q)\right\rangle_{\mathrm{e}}^{2}
$$

wherein $\langle\cdot\rangle_{\mathrm{e}}$ denotes the ensemble average over several scattering patterns. Thus, by calculating $\operatorname{var}\left(\hat{I}_{l}^{\mathrm{N}}(q)\right)$ from a scattering experiment information on the angular correlations can be obtained. Such functions based on angular correlations can be used to learn more about the symmetry of the underlying structures even if this is not directly obvious in a single scattering pattern $[6,8,10-13,17,20-22]$.

The orientational correlation function $\Psi_{l}(q)$ is determined from the USALS patterns from different films of dried microsphere dispersions. These $\Psi_{l}(q)$ spectra are compared with the structural information obtained by optical microscopy. In the present study, we focus ourselves on the predominant hexagonal lattice structure that is formed in our system. We characterize the degree of hexagonal order by the size $\sigma$ and number of hexagonally ordered domains of particles.

\section{EXPERIMENTAL DETAILS}

\section{A. Ultra-small-angle light-scattering setup}

Light-scattering experiments were performed on films made from microspheres. Since the samples exhibit scattering patterns at small scattering angles, an experimental setup with a two-dimensional camera for detection was employed. This type of ultra-small-angle light-scattering setup was successfully employed previously [23-25]. The schematic setup is depicted in Fig. 2. The setup employs a solid-state laser of wavelength $\lambda=532.3 \mathrm{~nm}$ and power $P=200 \mathrm{~mW}$. (The optical components were purchased from Thorlabs, Inc.) To remove stray light two achromatic lenses (L1, L2) of focal length $f=100 \mathrm{~mm}$ and a pinhole (PH1) are used that are optically conjugated. The beam is focused down on the sample (S) by using an additional achromatic lens (L3, $f=500 \mathrm{~mm}$ ). A second pinhole (PH2) is used to eliminate remaining stray light. The transmitted beam is blocked by a semitransparent beamstop (BS). The beamstop consists of a strongly absorbing filter glued on a highly transparent filter. The scattering light is focused by a fourth achromatic lens (L4) on a complementary metal-oxide-semiconductor- (CMOS) based camera $(\mathrm{C}, 1280 \times 1024$ pixels, $5.2-\mu \mathrm{m}$ pixel size, Thorlabs, Inc.) used for detecting the scattering pattern. To protect the camera from the intense scattered laser light the incoming beam is attenuated by two sets of filter wheels (FW). In total a transmission of $0.001 \%$ was used.

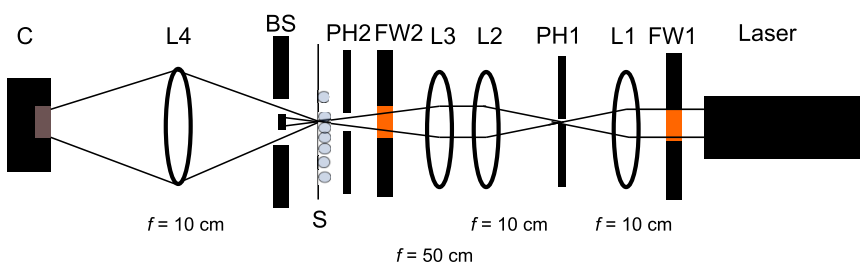

FIG. 2. (Color online) Scheme of the setup for ultra-small-angle light scattering. FW: filter wheel, L: lens, PH: pinhole, S: sample stage, BS: (semitransparent) beamstop, C: camera. 
The focus of the laser beam on the sample is $d_{\mathrm{L}}=300 \mu \mathrm{m}$. The sample stage consists of a motor stage that can move the sample perpendicular to the incoming beam and thus allows us to detect the scattering signal of different regions of the sample.

In order to relate the position of the scattering pattern on the camera to the corresponding scattering angle the diffraction signal of a pinhole with $200-\mu \mathrm{m}$ aperture is recorded beforehand. The shape of the wave front of the laser beam at the sample position is determined by a Hartmann-Shack wave-front sensor (Thorlabs, Inc.) with a resolution of $150 \mu \mathrm{m}$. The so-measured wave front was reconstructed using up to fourth-order Zernike polynomials [26].

\section{B. Samples and sample preparation}

For the USALS experiments Duke Standards microspheres and "particle size standards" silica were purchased from Thermo Scientific. The monodisperse microspheres consist of polystyrene particles dispersed in aqueous solution. The diameter of the microspheres are certificated by the supplier and are $D_{1}=(10.00 \pm 0.08) \mu \mathrm{m}$ (batch no. 4210-008), $D_{2}=$ $(12.01 \pm 0.11) \mu \mathrm{m}(4212-001)$, and $D_{3}=(14.97 \pm 0.10)$ $\mu \mathrm{m}$ (4215-005). The monodisperse silica particles in aqueous solution have a size of $D_{4}=(0.99 \pm 0.02) \mu \mathrm{m}(8100-003)$. In the following the particles are referred to as 10-, 12-, and $15-\mu \mathrm{m}$ microspheres and $1-\mu \mathrm{m}$ silicas, respectively.

Before use all particle dispersions were concentrated by centrifugating and pipetting the supernatant. From the concentrated dispersions mixtures of the $10-\mu \mathrm{m}$ microspheres with the 12- and $15-\mu \mathrm{m}$ microspheres as well as with two concentrations of the $1-\mu \mathrm{m}$ silicas were prepared. Equivalent volumes of the concentrated dispersions of the 10-, 12-, and $15-\mu \mathrm{m}$ microspheres and the $1-\mu \mathrm{m}$ silica were mixed. For the higher-concentrated $10-\mu \mathrm{m}$ microsphere $-1-\mu \mathrm{m}$ silica mixture, a 5-times-larger volume from the silica dispersion was used. The pure $10-\mu \mathrm{m}$-microsphere dispersion as well as the mixtures were dropped on a microscopy glass slide which

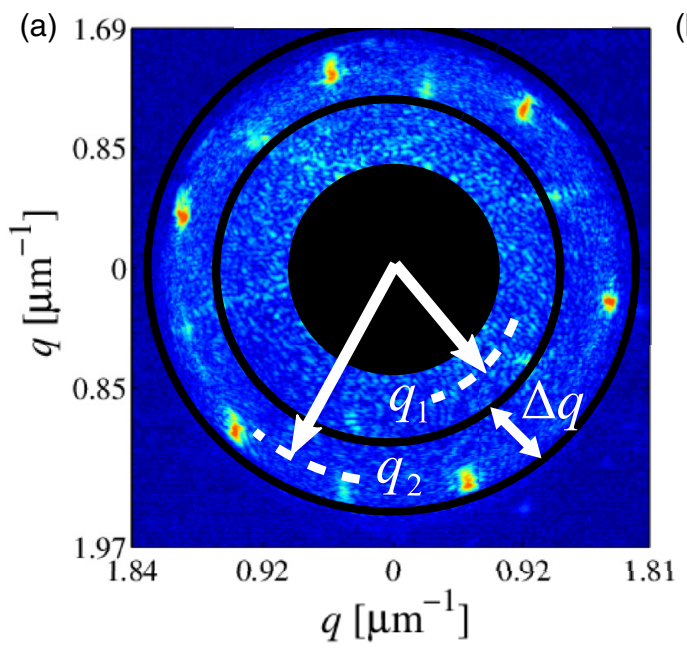

was slightly heated. After the solvent was evaporated, dried films formed in each case.

From these samples, light-scattering patterns were taken in transmission at different positions to employ the crosscorrelation analysis described in Sec. II. Therefore, the samples were placed on the motor stage in the laser focus and moved horizontally with a velocity of $0.445 \mathrm{~mm} / \mathrm{s}$ for a distance of about $20-25 \mathrm{~mm}$. The corresponding scattering patterns were recorded continuously by the camera. For each frame of the resulting film a region of interest was chosen that contained the central scattering signal. From these patterns dark images were subtracted. By this procedure about 300-500 scattering images were taken for each sample. From these scattering patterns the orientational correlation function $\Psi_{l}(q)$ was determined for the microsphere samples. The average error of the different Fourier components is obtained by analyzing the changes of $\Psi_{l}(q)$ with increasing number of patterns used for its computation. In more detail, plotting $\Psi_{l}(q)$ for a sufficiently large number of patterns gives the Fourier component fluctuating around its mean value. Taking the standard deviation of these gives the corresponding error.

In addition to the USALS measurements, several optical microscopy images were made from every microsphere film.

\section{RESULTS}

\section{A. Cross-correlation analysis on a two-dimensional semicrystalline microsphere film}

In Fig. 3(a) a representative scattering pattern of a film consisting solely of $10-\mu \mathrm{m}$ microspheres is shown. Therein, two broad $q$ partitions are chosen, for which the crosscorrelation analysis is performed. The broad increment of $\Delta q=0.57 \mu \mathrm{m}^{-1}$ had to be used for the following reasons. First, broad Bragg reflections are present that should be contained within the same $q$ partition. Second, due to the optics used, the scattering patterns are affected by aberrations. And, third, the position of the scattering patterns on the camera (b)

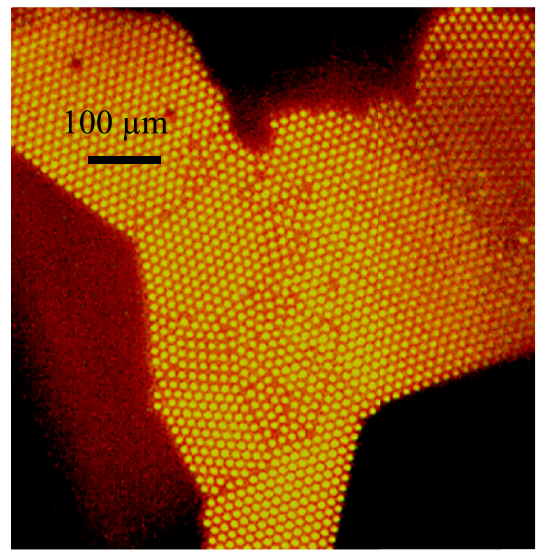

FIG. 3. (Color online) (a) Single scattering pattern of a monolayer film of 10- $\mu$ m polystyrene microspheres on a glass slide. Shown are the corresponding $q$ partitions used for the cross-correlation analysis centered at $q_{1}=0.86 \mu \mathrm{m}^{-1}$ and $q_{2}=1.44 \mu \mathrm{m}^{-1}$ and the increment $\Delta q=$ $0.57 \mu \mathrm{m}^{-1}$. (b) Micrograph of the sample. The presence of a monolayer film is clearly visible. The solid bar indicates a $100-\mu \mathrm{m}$ length scale. 
changes when the sample is moved as the intersection between laser focus and domains made of microspheres change.

In the scattering pattern diffraction spots are visible reflecting the semicrystalline film structure. In more detail, there are six highly intense Bragg spots equidistant from the beam center contained in $q_{2}$ partition centered at $1.44 \mu \mathrm{m}^{-1}$. Besides those intense six additional peaks of lower intensity are visible in the same $q$ range. In contrast, at lower scattering angles contained in $q_{1}=0.86 \mu \mathrm{m}^{-1}$, no Bragg peaks are directly visible and the scattering pattern looks more diffuse.

Inspecting the film by optical microscopy reveals that the microspheres form a monolayer with patches of the size of several particle diameters that have a hexagonal structure [Fig. 3(b)]. Thus, the scattering pattern can be understood by attributing the Bragg reflections to the two-dimensional hexagonal lattice.

The diffraction condition for a two-dimensional hexagonal lattice made out of spherical particles of diameter $D$ is given by $[27,28]$

$$
\sin \Theta_{(h, k)}=\frac{2}{\sqrt{3}} \frac{\lambda}{D n} \sqrt{h^{2}+k^{2}+h k},
$$

wherein $n$ denotes the index of refraction of the colloids. Thus, Bragg reflections of index $(h, k)$ are detectable at wave vectors of $q_{(h, k)}=4 \pi n / \lambda \sin \left(\Theta_{(h, k)} / 2\right)$.

For the lowest order of Bragg reflections of this structure $((1,0),(0,1),(\overline{1}, 0),(0, \overline{1}),(1, \overline{1}),(\overline{1}, 1))$, six reflections are expected for $q=0.73 \mu \mathrm{m}^{-1}$. This $q$ value is contained in the $q_{1}$ partition that covers the range $0.57 \mu \mathrm{m}^{-1} \leqslant q<$ $1.15 \mu \mathrm{m}^{-1}$. These reflections, however, are only barely visible in the experimental data [Fig. 3(a)]. The next two orders of Bragg spots $((2, \overline{1}),(1, \overline{2}),(\overline{2}, 1),(\overline{1}, 2),(1,1),(\overline{1}, \overline{1}))$ and $((2,0),(0,2),(\overline{2}, 0),(0, \overline{2}),(\overline{2}, 2),(2, \overline{2}))$ are located at $q=$ $1.26 \mu \mathrm{m}^{-1}$ and $q=1.45 \mu \mathrm{m}^{-1}$, respectively. Both types of reflections are contained within the $q_{2}$ partition covering $1.15 \mu \mathrm{m}^{-1} \leqslant q<1.73 \mu \mathrm{m}^{-1}$ and thus used together for the cross-correlation analysis.

Moving the sample through the laser beam results in 500 different diffraction patterns which have been used for the cross-correlation analysis. The resulting orientational correlation function for the different Fourier components, $\Psi_{l}(q)$, is shown in Fig. 4 for $l=5$ to 30 .

Scattering on the edges of the semicrystalline microsphere layer leads to streaks in the detected diffraction patterns. The analysis shows that this additional, parasitic signal leads to strong contributions of $l=2$ and $l=4$ in the CCA result. As this cannot simply be subtracted these contributions to $\Psi_{l}(q)$ are not considered further. The orientational correlation function $\Psi_{l}(q)$ displays also significant contributions from odd values of the Fourier components. Such odd values are forbidden for plane-wave illumination which always yields $I(q)=I(-q)$. However, deviations from an ideal plane wave illumination give rise also to odd values of $l$ (for details see Sec. VI).

For the $q_{1}$ partition centered at $0.86 \mu \mathrm{m}^{-1}$ the dominant value of $\Psi_{l}(q)$ is $l=6$. This peak at $l=6$ reflects the sixfold symmetry of the scattering pattern of the hexagonal lattice [19]. Thus, although the corresponding Bragg reflections are barely visible in the scattering patterns the underlying symmetry is revealed by the cross-correlation analysis.
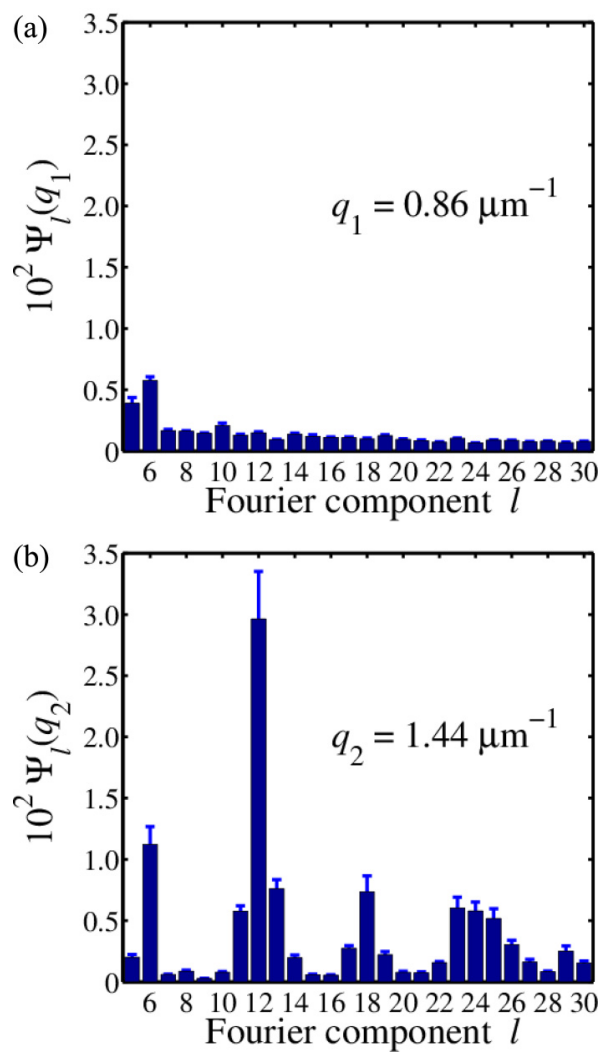

FIG. 4. (Color online) $\Psi_{l}(q)$ of the measured scattering patterns from the $10-\mu \mathrm{m}$-microsphere film as a function of the Fourier component $l$ for the two $q$ partitions. (a) $q_{1}$ centered at $0.86 \mu \mathrm{m}^{-1}$. (b) $q_{2}$ centered at $1.44 \mu \mathrm{m}^{-1}$.

The cross-correlation analysis at $q_{2}$ yields sharp peaks at $l=6, l=12$, and $l=18$ and a broad peak centered at $l=$ 24. Such a $\Psi_{l}(q)$ spectrum is characteristic for the hexagonal structure. Pronounced $l=6,12,18$, and 24 contributions are present for $q$ values close to the two types of Bragg spots as was shown both theoretically and by simulations for CCA on scattering patterns from single hexagons [19]. In the study by Lehmkühler et al. peaks at $l=6$ and 18 were found at the $q$ positions of the Bragg peaks, whereas peaks at $l=12$ and 24 are present for $q$ values between the reflections. In the present study on microsphere films with hexagonal lattice structure, all $l$ peaks are contained in the same $q$ partition for reasons discussed above.

The sixfold reflections at $q=1.26 \mu \mathrm{m}^{-1}$ and $q=$ $1.45 \mu \mathrm{m}^{-1}$ give rise to the prominent $l=6$ contribution in the orientational correlation function for the $q_{2}$ partition. The $l=12$ peak in the CCA spectrum reflects the fact that the two types of Bragg peaks are rotated with $30^{\circ}$ to each other (see insets in Figs. 3 and 5). The peaks at $l=18$ and 24 reflect higher harmonics of the pronounced peaks [19]. The broadening of the $l=24$ peak is probably due to the wave-front distortion of the incident laser beam.

Several microscopy images were taken from the sample from different spots showing all the presence of a semicrystalline hexagonal structure. The exact regions from which the diffraction patterns were detected could not be identified. Therefore, every image was subdivided into cutouts 

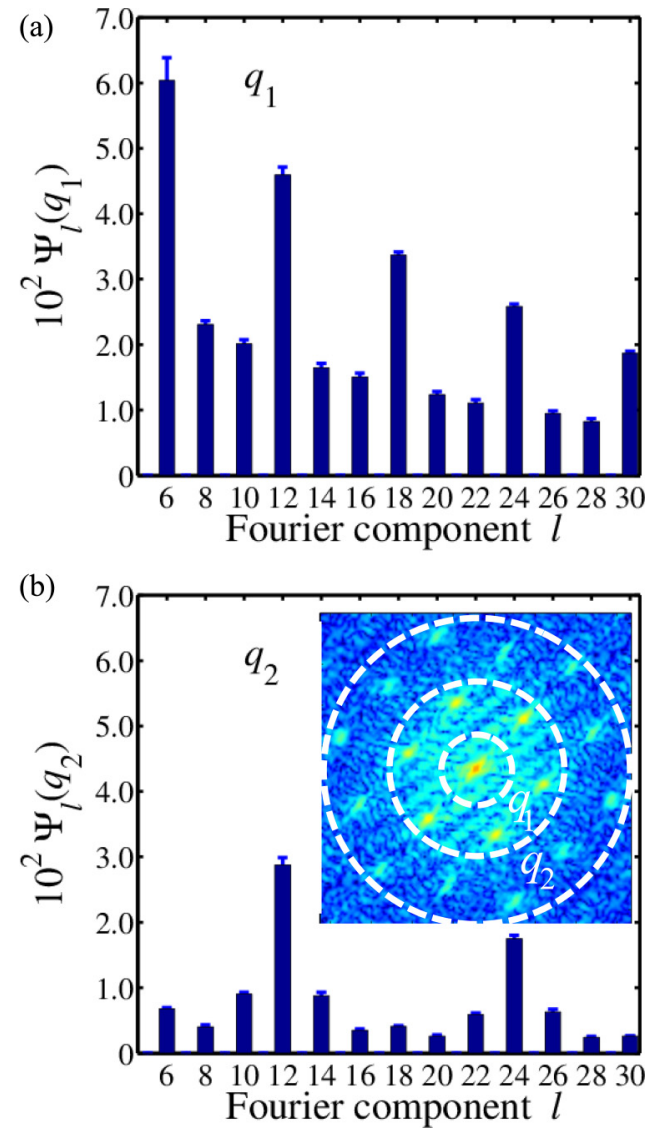

FIG. 5. (Color online) Results of the analysis of the calculated scattering patterns from the microscopy images of the $10-\mu \mathrm{m}$ microsphere film. $\Psi_{l}(q)$ for (a) $q_{1}$ and (b) $q_{2}$ assuming an undistorted wave front. The inset shows an example of the calculated scattering signal together with the respective $q$ partitions used for the CCA. It is visible that the two sets of Bragg reflexes contained in the $q_{2}$ partition are rotated with $30^{\circ}$ to each other.

of $300 \times 300 \mu \mathrm{m}$ size which is the size of the laser focus. The corresponding scattering patterns were subsequently calculated by Fourier transformation of these cutouts using a Hamming window function for noise suppression. With this approach the scanning of the sample with the laser beam is simulated.

An example of such a calculation is given in the inset in Fig. 5(b). Herein, the different, relevant Bragg reflections of the two-dimensional hexagonal lattice are visible. Using these calculated scattering patterns, the same cross-correlation analysis was performed as for the experimental data. The results of this for both $q$ partitions is given in Figs. 5(a) and $5(\mathrm{~b})$

For the $q_{1}$ partition there is a pronounced $l=6$ peak in the $\Psi_{l}(q)$ spectrum. This is similar for the $q_{2}$ partition where $l=$ 12 is highlighted. For both $q$ partitions, additional maxima of $\Psi_{l}(q)$ are present. These are located at $l=12, l=18$, and $l=24$. These are the aforementioned higher harmonic contributions of the CCA [19].

Comparing the results from CCA of the measured and the calculated scattering patterns shows that in both spectra the maximum of $\Psi_{l}(q)$ is present for the same $l$ component.
Differences of the form of the spectra are due to the presence of a nonplanar wave front. The different amplitudes of the $\Psi_{l}(q)$ components is attributed to the simple approach of simulating scattering patterns by calculation of Fourier transform from micrograph cutouts. A direct comparison of the absolute values is only possible between the CCA spectra obtained either from the USALS measurement or by calculation from the microscopy images.

\section{B. LCCA on disordered microsphere films}

In a next step, different mixtures of microspheres have been prepared and studied by light scattering and cross-correlation analysis as well as by optical microscopy (Fig. 6). The intention to mix different microspheres was to reduce the number and size of hexagonal domains formed by the $10-\mu \mathrm{m}$ particle and to study via CCA how this affects the orientational correlation function $\Psi_{l}(q)$. The focus is on those $l$ values that indicate the hexagonal order, i.e., $l=6$ and 12. The full list of samples studied is given in Table I. For every sample several micrographs were taken. From these, the average correlation length $\sigma$ of the hexagonal domains was determined by counting the number of ordered microspheres within a domain. The USALS measurements have been performed in different experimental campaigns. Thus, the sample-detector-distance was not exactly the same for all samples, which led to slightly different positions on the camera. This was taken into account when performing the LCCA.

Sample A is the already-discussed monolayer film made up solely of 10- $\mu \mathrm{m}$ microspheres [Fig. 6(a)]. The large hexagonal domains with correlation length of $\sigma=(346 \pm 79) \mu \mathrm{m}$ give rise to pronounced Bragg reflections. The average domain size is larger than the diameter of the laser beam. The CCA spectrum at $q_{1}$ with the pronounced $l=6$ contribution is shown as well.

Sample B consists of a mixture of 10 - and $15-\mu \mathrm{m}$ microspheres [Fig. 6(b)]. When scanning the sample different regions are illuminated. Some of these give rise to USALS patterns showing Bragg reflections, whereas others display Debye-Scherrer powder diffraction rings. In addition, there are regions where both scattering signals add up. Due to the different arrangement of the sample and the camera and the much broader rings, the first two diffraction rings are detectable for this sample. The amplitude of the CCA spectrum for the $l$ values under discussion obtained from the different scattering patterns is reduced compared to sample A. Focusing on the hexagonal symmetry indicated by $l=6$ for the $q_{1}$ partition, the CCA result indicates that the underlying order is reduced. Although not every frame taken from the sample showed distingued Bragg peaks, there is a dominant peak in the CCA spectrum for $l=6$. From the micrographs the actual structure of the monolayer film was determined. The different radii of the microspheres led to a partial reduction of the size $[\sigma=(180 \pm 44) \mu \mathrm{m}]$ and number of ordered domains made of the smaller particle species. The larger microspheres do not form domains but rather induce a distortion of hexagonal structure of the $10-\mu \mathrm{m}$-sphere film as can be seen from the microscopy images. This is reflected in the USALS patterns. Thus, the reduced values for $\Psi_{l}(q)$ are reflecting the more random structure within the mixture. 

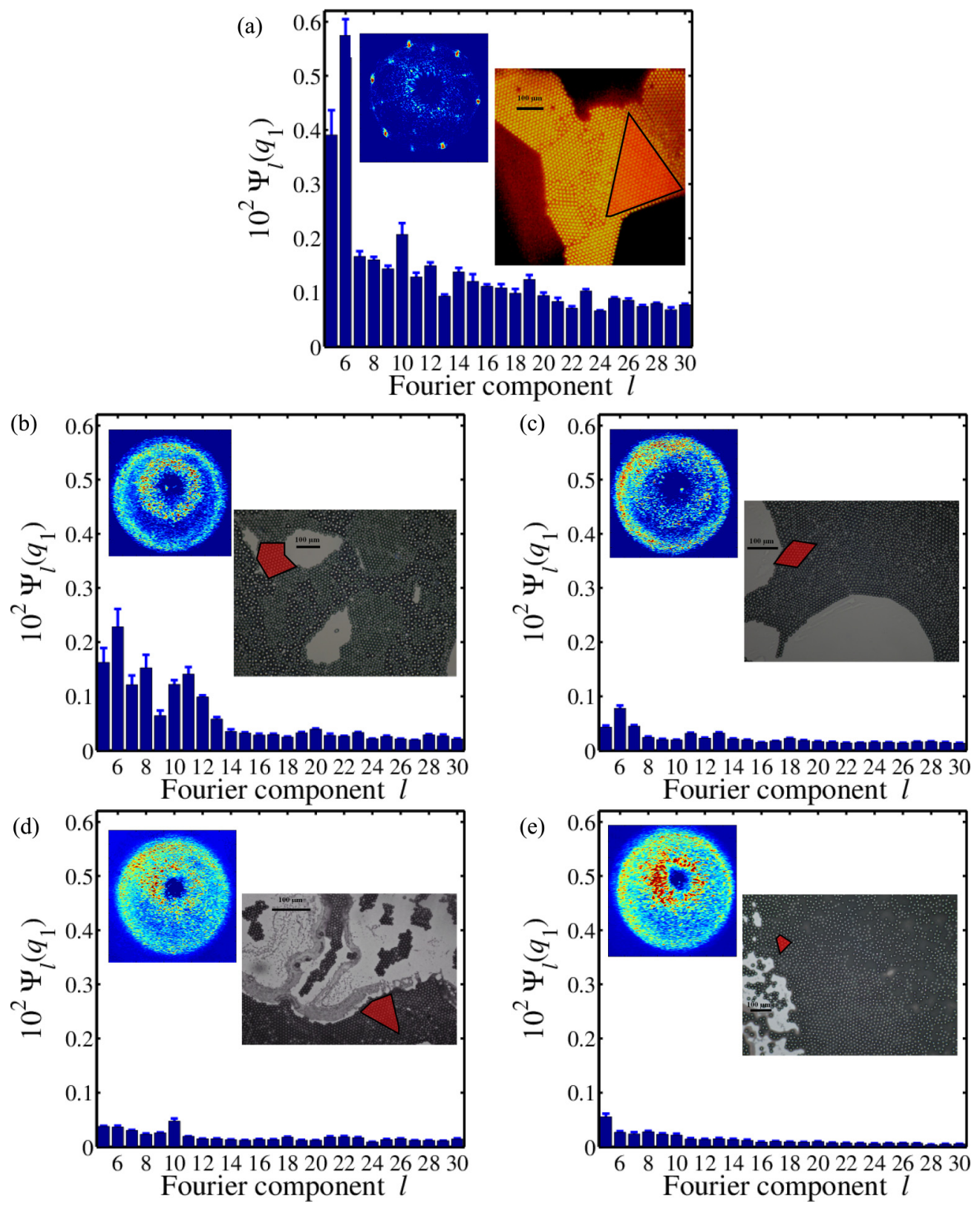

FIG. 6. (Color online) Results of the cross-correlation analysis at $q_{1}$ for (a) the pure 10- $\mu \mathrm{m}$ microspheres on a glass slide and for different mixtures of 10- $\mu \mathrm{m}$ microspheres with other particles [(b) 15- $\mu \mathrm{m}$ microspheres, (c) 12- $\mu \mathrm{m}$ microspheres, and (d) low- and (e) high-concentrated $1-\mu \mathrm{m}$ silicas]. In the insets, typical scattering patterns as well as microscopic images are shown. A typical domain of correlated particles of size $\sigma$ is highlighted.

When mixing the $10-\mu \mathrm{m}$ microspheres with the $12-\mu \mathrm{m}$ ones (sample C), the USALS patterns exhibit a diffuse diffraction ring [Fig. 6(c)]. This indicates, compared to sample $\mathrm{B}$, a stronger perturbation of the underlying microsphere film. The amplitude of $\Psi_{l}(q)$ is reduced with respect to the previously discussed sample. However, the $l=6$ peak is still visible. Inspecting the sample with a microscope shows that the two types of microspheres are phase separated. At this size fraction $(\eta=0.83)$ both the $10-\mu \mathrm{m}$ microspheres and the $12-\mu \mathrm{m}$ microspheres form independent hexagonal domains with a size $\sigma=(169 \pm 31) \mu \mathrm{m}$ that are separated by disordered regions. Mixing the microspheres reduces the degree of hexagonal order in the sample as $\sigma$ is smaller than that of the previously discussed samples. This disorder of the hexagonal lattice structure is reflected in the scattering patterns and by the CCA spectrum.

Sample D is a mixture of the $10-\mu \mathrm{m}$ microspheres with $1-\mu \mathrm{m}$ silica spheres [Fig. 6(d)]. For this sample the recorded USALS patterns show only a strong signal close to the primary beam which is most probable due to stray light from the sample and not used for the cross-correlation analysis. No Bragg peaks or pronounced Debye-Scherrer rings are present. The average value of the corresponding CCA spectrum is similar to that of sample $\mathrm{C}$, however, lacking the peak at $l=6$. In contrast, a peak is present at $l=10$. This might stem from local pentagonal domains in the sample as that 
TABLE I. List of the dried microsphere films studied. Given are the sample composition, the ratio $\eta$ of particle sizes, and the domain size $\sigma$ obtained from the micrographs.

\begin{tabular}{lccc}
\hline \hline Sample name & Composition & Size ratio $\eta$ & Domain size $\sigma(\mu \mathrm{m})$ \\
\hline $\mathrm{A}$ & $10 \mu \mathrm{m}$ & 1 & $346 \pm 79$ \\
$\mathrm{~B}$ & $10 \mu \mathrm{m}+15 \mu \mathrm{m}$ & 0.67 & $180 \pm 44$ \\
$\mathrm{C}$ & $10 \mu \mathrm{m}+12 \mu \mathrm{m}$ & 0.83 & $169 \pm 31$ \\
$\mathrm{D}$ & $10 \mu \mathrm{m}+1 \mu \mathrm{m}$ & 0.10 & $100 \pm 25$ \\
$\mathrm{E}$ & $10 \mu \mathrm{m}+1 \mu \mathrm{m}$ & 0.10 & $80 \pm 21$ \\
\hline \hline
\end{tabular}

would give rise to stronger $l=10$ contributions [19]. However, from the micrographs only rarely single pentagonal domains located close the microsphere film are visible. The $1-\mu \mathrm{m}$ silica spheres do not form a monolayer but mulitlayered regions that look opaque as can be seen from the micrographs. Thus, the scattering patterns might be as well affected by multiply scattering contributions. The areas made out of silica particles separate the hexagonal microsphere structures which leads to a reduced correlation length $[\sigma=(100 \pm 25) \mu \mathrm{m}]$.

Increasing the concentration of silica particles in the mixture with the 10- $\mu \mathrm{m}$ microspheres (sample E) only slightly affects the USALS pattern in comparison to sample D [Fig. 6(e)]. Only in the region close to the beamstop, which is most probably corrupted by stray light, stronger intensity is recorded. The corresponding CCA spectrum is void of any characteristic peaks. The small $l=5$ contribution is most probably an artifact from scattering from the silica multilayers. The amount and size of these is increased for the higher silica concentration in comparison to sample D. The micrographs taken from this sample display small clusters of microspheres of correlation length $\sigma=(80 \pm 21) \mu \mathrm{m}$ that are immersed in a matrix of silicas which work as spacers between the larger particles.

By mixing the 10- $\mu \mathrm{m}$ microspheres with particles of different size the degree of hexagonal order of the particle film structure is changed, as is revealed by the microscopy images and by the USALS pattern. For a size ratio of $\eta=0.67$ (sample B) $10-\mu \mathrm{m}$ microspheres form ordered patches with the largest domain size of all mixtures studied. The $15-\mu \mathrm{m}$ microspheres form apparently only disordered domains. Increasing the ratio of radii to $\eta=0.83$ (sample $C$ ) leads to the formation of smaller domains for both types of microspheres (10 $\mu \mathrm{m}$ and $12 \mu \mathrm{m})$. Interestingly, for two-dimensional binary particle films with particle size ratios of $\eta=0.76$ and 0.86 an amorphous phase was reported in the literature, whereas for ratios of $\eta=0.87$ and 0.90 ordered hexagonal structures were observed [29,30]. This is similar to our results for sample $\mathrm{C}$ for that $\eta$ is within this values. Differences in the actual type of structures can be attributed to the different fraction of particles within the mixtures. For binary colloidal mixtures confined between two glass plates at $\eta=0.11$ and 0.22 a transition from a solid to a liquid phase structure is reported which depends on the mixing ratio [31]. For smaller size ratios $\eta=0.025$ and 0.05 this confined system displays phase separation of the two particle species. If the confinement, ensuring monolayer formation, is absent, phase separation of particles sets in at a higher size ratio $(\eta=0.29)$ as was reported for a layer of dried colloids [32].
This results in ordered structures made from larger colloids surrounded by partially ordered rings of the smaller species around them. This is similar to our results for sample $\mathrm{E}$ ( $\eta=$ $0.10)$. For lower concentrations of $1-\mu \mathrm{m}$ silicas in the mixture, a more ordered structure is present (sample D) as is shown by the micrographs.

\section{COMPARISON BETWEEN THE MAGNITUDES OF THE $L=6$ AND 12 CONTRIBUTIONS AND THE SIZE OF HEXAGONAL DOMAINS}

The analysis of the mixtures shows that the magnitude of the $l=6$ contributions in the CCA spectra depends on the degree of hexagonal order, i.e., number and size of ordered domains, of the respective film. In the following we compare the $l=6$ component of the orientational correlation function with the average correlation length normalized to the size of the illuminated area $\left(\sigma / d_{\mathrm{L}}\right)$ for samples A-E.

Figure 7(a) displays the magnitude of the $l=6$ component of the orientational correlation function at the $q_{1}$ partition for the mixtures and the pure $10-\mu \mathrm{m}$-microsphere film plotted as a function of $\sigma / d_{\mathrm{L}}$. Sample $\mathrm{E}$ with the smallest domain size exhibits the lowest magnitude of the $l=6$ Fourier component of all the samples studied. As the domain size increases for the other samples, we find that the $l=6$ component increases as well. Similar findings are present for the CCA spectrum at the $q_{2}$ partition [Fig. 7(b)], wherein $\Psi_{l}(q)$ is displayed for $l=6$ [blue (dark gray) filled squares] and
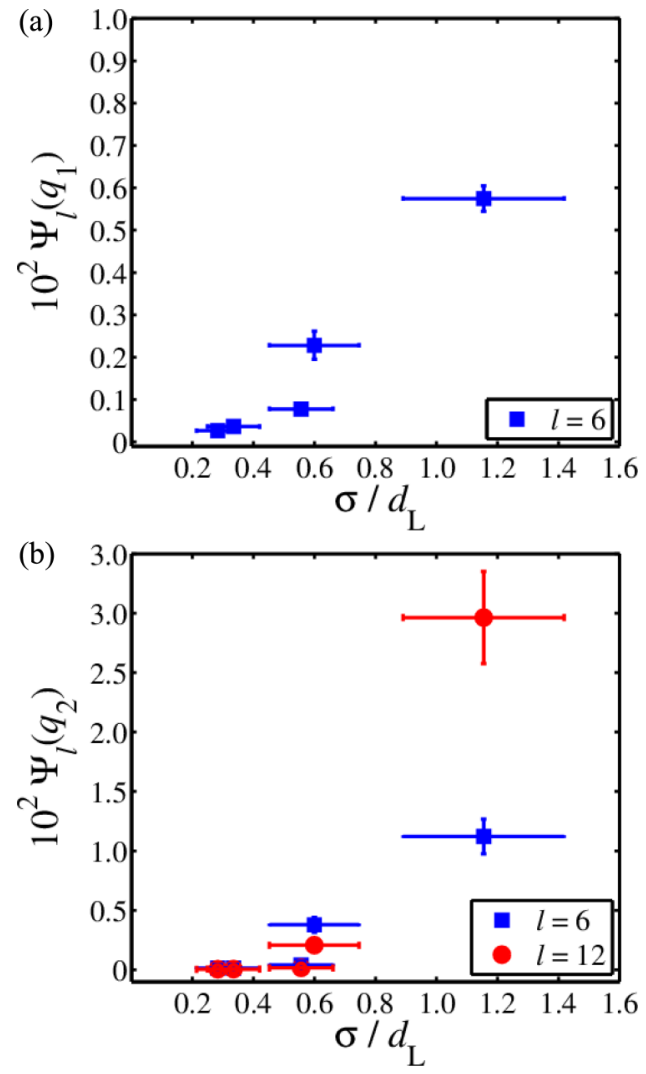

FIG. 7. (Color online) The dependence of $\Psi_{l}(q)$ on the correlation length divided by the size of the laser beam, $\sigma / d_{\mathrm{L}}$, for (a) $l=6$ [blue (dark gray) filled squares] at $q_{1}$ and (b) $l=6$ [blue (dark gray) filled squares] and $l=12$ [red (light gray) filled circles] at $q_{2}$. 

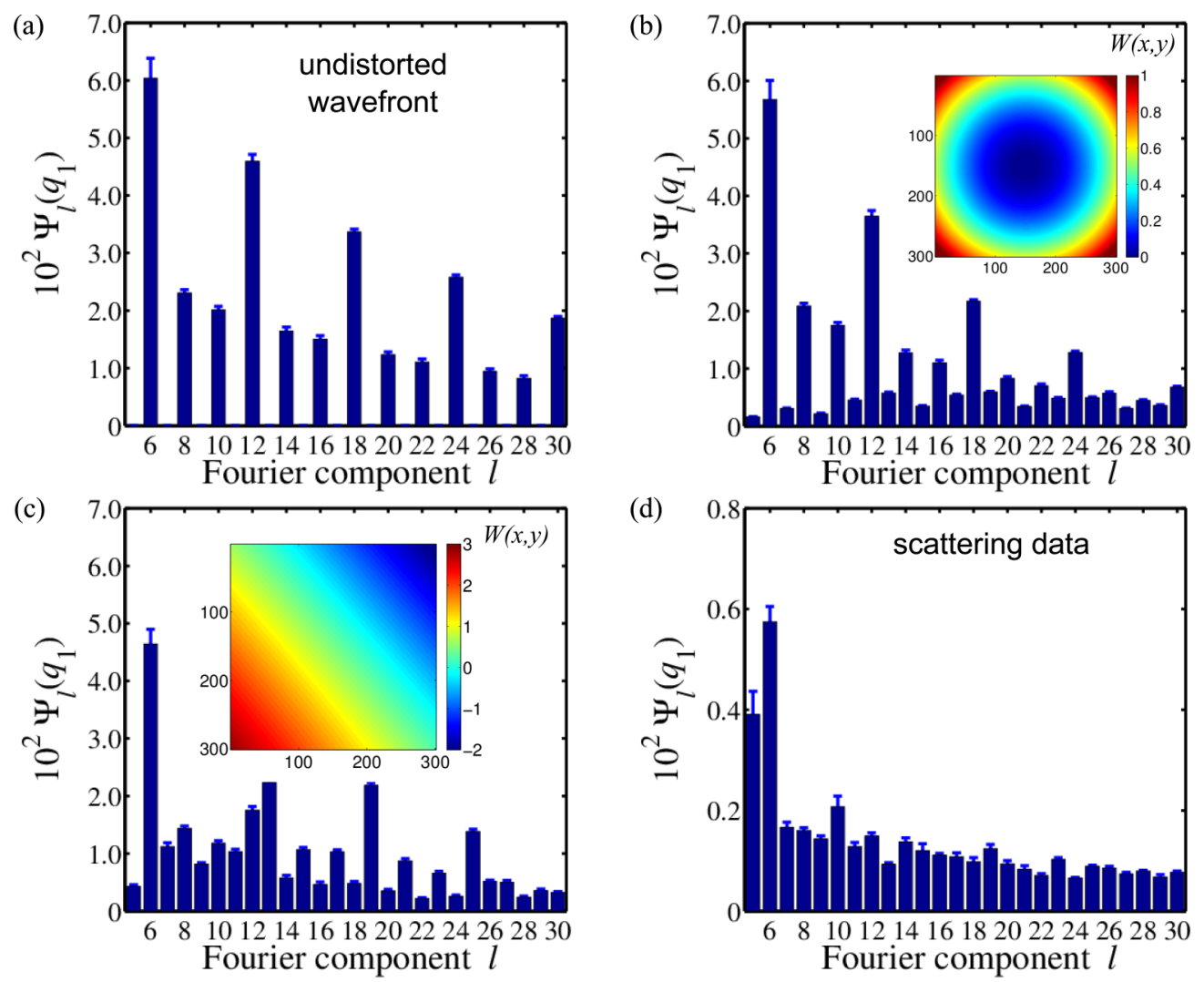

FIG. 8. (Color online) The influence of the wave front on $\Psi_{l}(q)$ obtained from the microscopy images of the pure 10- $\mu \mathrm{m}-\mathrm{microsphere}$ monolayer for $q_{1}$ : (a) for a plane wave, (b) a parabolic profile with $A=3 \times 10^{-4}$, and (c) the experimentally determined wave front of the USALS setup. (d) CCA spectrum from the scattering patterns. The insets show on a $\mu \mathrm{m}$ scale the wave front $W(x, y)$ used.

$l=12$ [red (light gray) filled circles]. Here the magnitudes of the $l$ components characterizing the hexagonal lattice also increase when the domain size becomes larger. Notably, the possible contributions of multiple scattering present for samples D and E might lead to even more reduced $l=6$ and 12 Fourier components.

The result of this comparison suggests that there is a correlation between the amplitude of $l=6$ and 12 of the components of $\Psi_{l}(q)$ and the size of the hexagonal domains. For domains whose sizes are smaller than the laser beam diameter there is an increase of the $\Psi_{6}(q)$ and $\Psi_{12}(q)$ value as $\sigma$ becomes larger. This can be qualitatively understood by considering the ratio of the average domain size $\sigma$ and beam diameter $d_{\mathrm{L}}[33,34]$.

For $\sigma / d_{\mathrm{L}} \ll 1$, different small hexagonal domains are illuminated on a single position that give rise to a diffuse scattering signal. When moving the sample and detecting the scattering from another position, USALS patterns of similar types of domains are recorded. As these detected scattering patterns and thus $\hat{I}_{l}^{\mathrm{N}}(q)$ for $l=6$ and 12 are similar under this condition, the change in $\Psi_{l}(q)$ is, as expected, small. For larger $\sigma / d_{\mathrm{L}}$ values, the differences between USALS patterns will be more pronounced, which should be reflected by an increase of the relevant Fourier components in $\Psi_{l}(q)$.

Based on this simple model, we expect the strongest contribution of the $l=6$ and 12 values to $\Psi_{l}(q)$ to be present if $\sigma / d_{\mathrm{L}} \approx 1$. Under this condition the average domain size is similar to the beam size.

\section{THE INFLUENCE OF THE WAVE-FRONT DISTORTION}

In the experimental CCA spectra, odd values of $l$ are present which are absent in the spectra obtained from the diffraction patterns calculated from the microscopy images [Fig. 8(a)]. In order to investigate the origin of these odd number components, we analyzed the effect of a distorted wave front on the cross-correlation-analysis results.

As the wave front in the USALS experiment is not plane, aberrations have to be taken into account. This wave-front distortion $W(x, y)$ induces a nonuniform phase factor $\Phi(x, y)$ [35]

$$
\Phi(x, y)=\frac{2 \pi}{\lambda} W(x, y) .
$$

In order to simulate this effect on the CCA spectra, $\Phi(x, y)$ is introduced into the Fourier transformation FT of the cutouts $R(x, y)$ of the microscopy images from the pure $10-\mu \mathrm{m}-$ microsphere film,

$$
I\left(q_{x}, q_{y}\right)=|\mathrm{FT}(R(x, y) \exp (i \Phi(x, y)))|^{2} .
$$

As the first and simplest choice, a parabolic wave front of amplitude $a$ is assumed,

$$
W(x, y)=a\left(x^{2}+y^{2}\right) .
$$

The result of the cross-correlation analysis of the so-obtained scattering patterns is displayed in Fig. 8(b) for $A=(2 \pi / \lambda) a=$ 
$3 \times 10^{-4}$. Switching on the phase factor directly results in the presence of odd number components in the CCA spectrum.

In a next step, the actual wave front of the incident light beam at the sample position was measured using a wave-front sensor [inset in Fig. 8(c)]. The CCA spectrum calculated from the exact wave front is given in Fig. 8(c). The real aberrations lead to smaller values of $\Psi_{l}(q)$ as well as to a broadening of the characteristic value around $l=6$. The corresponding CCA spectrum resembles the one obtained from the light-scattering data [Fig. 8(d)].

We assume that the differences between these two stem from the fact that the determination of the wave front is limited by the resolution $\Delta x$ of the wave-front sensor. As $\Delta x=$ $150 \mu \mathrm{m}$ is within the size of the laser focus $\left(d_{\mathrm{L}}=300 \mu \mathrm{m}\right)$, the actual light front might deviate from the reconstructed one. Therefore, we assume that the effect of wave-front distortion obtained with the Hartmann-Shack sensor on the LCCA spectra is overestimated compared to the measured scattering data. Moreover, the optics after the sample, i.e., the beamstop and the additional lens focusing on the camera, might affect the scattering pattern as well. Notably, the contribution of the form factor of the microspheres to the scattering patterns has no effect on the CCA spectrum and does not need to be taken into account.

The presented analysis shows that the differences between the CCA spectra determined from the USALS experiment and the microscopy images can be explained by the nonplanar wave front $W(x, y)$. Including this in the calculations of the scattering patterns from the micrograph cutouts, general features of $\Psi_{l}(q)$ determined from the USALS data can be simulated.

\section{CONCLUSION}

We have performed an USALS study on different twodimensional monolayers of microspheres and analyzed the scattering patterns with the same cross-correlation-analysis scheme as used for XCCA $[6,19]$. The results were compared to CCA spectra of calculated scattering patterns from microscopy images. The micrographs directly show the structure of the film and the size of ordered domains. With this combined approach we were able to directly relate the results of the cross-correlation analysis to the underlying structure of the film.

For a pure $10-\mu \mathrm{m}$-microsphere film defined Bragg spots were present for large $q$ ( $q_{2}$ partition) that reflect the hexagonal structure that is directly visible with the microscope. LCCA reveals the hexagonal structure via the presence of strong $l=$ 6 and $l=12$ contributions in the orientational correlation function $\Psi_{l}(q)$. Notably, for the $q_{1}$ partition, the scattering pattern looks diffuse but a pronounced $l=6$ contribution is visible in the LCCA. This shows that the LCCA scheme reflects orientational order that is not directly visible from the scattering pattern. The CCA spectrum extracted from the calculated USALS patterns using the micrograph cutouts exhibits both similarities [maximum of $\Psi_{l}(q)$ present for the same $l$ component] as well as differences to the experimental data. It is shown that the presence of odd $l$ values and the peak broadening in the experimental spectra stems for the nonplanar wave front of the laser beam.

Mixtures of differently sized microspheres were also studied. Due to the mixing the number and size of two-dimensional hexagonal ordered domains was systematically reduced, as confirmed by the microscopy images. This decrease of hexagonal order resulted in the absence of Bragg reflections in the scattering patterns. Only Debye-Scherrer rings and more diffuse patterns were visible.

These USALS experiments on two-dimensional samples showed that the magnitude of $l=6$ and $l=12$ contributions to the orientational correlation function $\Psi_{l}(q)$ increased with increasing size of the hexagonal domains. This indicates that the degree of hexagonal order can be directly quantified by the magnitude of the $l=6$ and 12 contributions of $\Psi_{l}(q)$.

By analyzing the results of the CCA for calculated patterns from the micrographs it was found that only even Fourier components $l$ are present as predicted from theory. The USALS measurements show that odd numbers are present as well. This experimental finding is due to the distortions of the wave front of the laser light. By introducing a phase factor, odd numbers could be generated in the CCA spectra. This distortion leads to the broadening of the peaks in $\Psi_{l}(q)$ as was demonstrated using the measured aberrations.

In summary, we have shown that using angular crosscorrelation analysis of several independent scattering patterns allows to study the orientational order in a two-dimensional sample. The orientational correlation function $\Psi_{l}(q)$ was shown to quantitatively describe the degree of order of the twodimensional films consisting of hexagonal domains. Moreover, characteristic features in the CCA spectra could be attributed to the underlying sample structure and additional contributions of the wave-front distortion.

We argue that the findings reported herein for a twodimensional system will have an influence for the crosscorrelation analysis of three-dimensional systems. Future applications, especially at XFEL facilities [7], should largely benefit from these types of cross-correlation analysis as these will allow us to investigate the local order and even its evolution in putative disordered sample systems. For these types of experiments several requirements were discussed recently [36]. Our results suggest that beam size and the planarity of the wave front [37] are important experimental parameters.

\section{ACKNOWLEDGMENTS}

This work has been supported by the excellence cluster "The Hamburg Centre for Ultrafast Imaging - Structure, Dynamics, and Control of Matter at the Atomic Scale" of the DFG. The authors thank F. Lehmkühler for helpful discussions on the cross-correlation analysis and S. Schleitzer and W. Roseker for helpful discussions on the USALS setup.
[1] J. P. Hansen and I. R. McDonald, Theory of Simple Liquids (Academic Press, London, 1986).
[2] P. A. Egelstaff, An Introduction to the Liquid State (Clarendon Press, Oxford, 1992). 
[3] A. Zallen, The Physics of Amorphous Solids (Wiley-VCH, Weinheim, 1998).

[4] P. A. Egelstaff, D. I. Page, and C. R. T. Heard, J. Phys. C: Solid St. Phys. 4, 1453 (1971).

[5] M. A. Schroer, M. Tolan, and R. Winter, Phys. Chem. Chem. Phys. 14, 9486 (2012).

[6] P. Wochner, C. Gutt, T. Autenrieth, T. Demmer, V. Bugaev, A. Diaz Ortiz, A. Duri, F. Zontone, G. Grübel, and H. Dosch, Proc. Natl. Acad. Sci. USA 106, 11511 (2009).

[7] P. Emma et al., Nat. Photon. 4, 641 (2010).

[8] Z. Kam, Macromolecules 10, 927 (1977).

[9] D. K. Saldin, V. L. Shneerson, M. R. Howells, S. Marchesini, H. N. Chapman, M. Bogan, D. Shapiro, R. A. Kirian, U. Weierstall, K. E. Schmidt, and J. C. H. Spence, New J. Phys. 12, 035014 (2010).

[10] D. K. Saldin, H. C. Poon, M. J. Bogan, S. Marchesini, D. A. Shapiro, R. A. Kirian, U. Weierstall, and J. C. H. Spence, Phys. Rev. Lett. 106, 115501 (2011).

[11] G. Chen, M. A. Modestino, B. K. Poon, A. Schirotzek, S. Marchesini, R. A. Segalman, A. Hexemer, and P. H. Zwart, J. Synchrotron Rad. 19, 695 (2012).

[12] D. Starodub et al., Nat. Commun. 3, 1276 (2012).

[13] B. Pedrini, A. Menzel, M. Guizar-Sicairos, V. A. Guzenko, S. Gorelick, C. David, B. D. Patterson, and R. Abela, Nat. Commun. 4, 1647 (2013).

[14] N. A. Clark, B. J. Ackerson, and A. J. Hurd, Phys. Rev. Lett. 50, 1459 (1983).

[15] J. M. Gibson, M. M. J. Treacy, T. Sun, and N. J. Zaluzec, Phys. Rev. Lett. 105, 125504 (2010).

[16] P. Wochner, M. Castro-Colin, S. N. Bogle, and V. N. Bugaev, Int. J. Mat. Res. 102, 874 (2011).

[17] M. Altarelli, R. P. Kurta, and I. A. Vartanyants, Phys. Rev. B 82, 104207 (2010).

[18] R. P. Kurta, M. Altarelli, E. Weckert, and I. A. Vartanyants, Phys. Rev. B 85, 184204 (2012).

[19] F. Lehmkühler, G. Grübel, and C. Gutt, J. Appl. Cryst. (2014), doi:10.1107/S1600576714012424.
[20] Z. Kam, M. H. J. Koch, and J. Bordas, Proc. Natl. Acad. Sci. USA 78, 3559 (1981).

[21] R. A. Kirian, J. Phys. B 45, 223001 (2012).

[22] R. P. Kurta, B. I. Ostrovskii, A. Singer, O. Y. Gorobtsov, A. Shabalin, D. Dzhigaev, O. M. Yefanov, A. V. Zozulya, M. Sprung, and I. A. Vartanyants, Phys. Rev. E 88, 044501 (2013).

[23] L. Cipelletti and D. A. Weitz, Rev. Sci. Instrum. 70, 3214 (1999).

[24] K. Nishida, H. Ogawa, G. Matsuba, T. Konishi, and T. Kanaya, J. Appl. Cryst. 41, 723 (2008).

[25] D. Lee, I. A. Gutowski, A. E. Bailey, L. Rubatat, J. R. de Bruyn, and B. J. Frisken, Phys. Rev. E 83, 031401 (2011).

[26] D. Malacara, ed., Optical Shop Testing (John Wiley \& Sons, New York, 2007).

[27] I. M. Krieger and F. M. O’Neill, J. Am. Chem. Soc. 90, 3114 (1968).

[28] A. K. Samusev, K. B. Samusev, M. V. Rybin, M. F. Limonov, E. Y. Trofimova, D. A. Kurdyukov, and V. G. Golubev, Phys. Solid State 53, 1056 (2011).

[29] A. S. Nowick and S. R. Mader, IBM J. Res. Dev. 9, 358 (1965).

[30] D. R. Nelson, M. Rubinstein, and F. Spaepen, Philos. Mag. A 46, 105 (1982).

[31] B. Cui, B. Lin, and S. A. Rice, J. Chem. Phys. 119, 2386 (2003).

[32] E. Liniger and R. Rishi, J. Am. Ceram. Soc. 70, 843 (1987).

[33] L. Fan, D. Paterson, I. McNulty, M. M. J. Treacy, and J. M. Gibson, J. Microsc. 225, 41 (2007).

[34] A. T. J. Torrance, B. Abbey, C. T. Putkunz, D. Pelliccia, E. Balaur, G. J. Williams, D. J. Vine, A. Y. Nikulin, I. McNulty, H. M. Quiney, and K. A. Nugent, Opt. Express 21, 28019 (2013).

[35] M. Born and E. Wolf, eds., Principles of Optics (Cambridge University Press, Cambridge, 1999).

[36] R. A. Kirian, K. E. Schmidt, X. Wang, R. B. Doak, and J. C. H. Spence, Phys. Rev. E 84, 011921 (2011).

[37] S. Rutishauser, L. Samoylova, J. Krzywinski, O. Bunk, J. Grünert, H. Sinn, M. Cammarata, D. M. Fritz, and C. David, Nat. Comm. 3, 947 (2012). 